\title{
El seguro agrario como instrumento de estabilización de rentas. La experiencia española de finales del siglo $\mathbf{X X}$.
}

\author{
Agricultural insurance as an instrument of income stabilization. The \\ Spanish experience at the end of twentieth (20th) century.
}

\author{
Santiago Escribano Pintor* \\ Departamento de Economía y Ciencias Sociales Agrarias, Escuela Técnica Superior de Ingenieros Agrónomos, Universidad Politécni- \\ ca de Madrid, Madrid, España. \\ Autor para correspondencia: sescriba@pas.ucm.es
}

Manuscrito recibido el 22 de abril de 2010.Aceptado, tras revisión, el 15 de julio de 2010

\section{Resumen.}

La importancia del seguro agrario, como instrumento de política agraria, en cuanto al mantenimiento de rentas de los pequeños y medianos agricultores, sostén de la agricultura tradicional y respetuosa con el medio ambiente, podemos observarla en el caso español. En este país, desde 1978 se legisló acertadamente sobre este tema, siendo actualmente el modelo español de seguros agrarios un ejemplo a seguir por numerosos países al haber conseguido llegar a un equilibrio entre las diversas partes interesadas y lograr un adecuado mantenimiento de las rentas de los agricultores cuando sus cosechas se encuentran siniestradas por fenómenos meteorológicos.

Palabras clave: seguros agrarios, rentas agrarias, siniestros, España.

\begin{abstract}
The relevance of agricultural insurance as an instrument of agricultural policy for the income maintenance of small and medium-sized farmers, support of traditional agriculture and respect of the environment, can be observed in Spain. In this country the subject was legislated correctly since 1978. The Spanish agricultural insurance model became an example to follow by many countries inasmuch as it attained the balance between the parties involved and achieved proper income maintenance for farmers in case of crop failures by meteorological phenomena.
\end{abstract}

Keywords: Agricultural insurance, agricultural income, disasters, Spain.

Forma sugerida de citar:

Escribano, S. El seguro agrario como instrumento de estabilización de rentas. La experiencia española de finales del siglo XX. La Granja Vol.I I(I). Pp I5-2I 


\section{Introducción.}

Actualmente consideramos a la sociedad española como una sociedad moderna y avanzada que progresa hacia cotas de desarrollo insospechadas, sobre todo si la comparamos con la de hace unas décadas. Como consecuencia de lo anterior, un signo inequívoco de dicho avance lo encontramos en la capacidad de dotarse de unos sistemas de protección y garantía que logran reducir el riesgo en el desempeño de las distintas actividades productivas. Por tanto, el sector agrario no puede arrinconarse en posturas primitivas de evasión de dicha realidad, máxime cuando además existe un amplio consenso, entre las diferentes instituciones y personas comprometidas con este sector, en considerar los seguros agrarios como un importante mecanismo de seguridad, cuya preservación y buen funcionamiento constituye una garantía de seguridad imprescindible, no sólo para los propios agricultores, sino para el resto de la sociedad (López de Coca, 1997). Dentro de las principales investigaciones que se han desarrollado sobre este tema, nos encontramos con Trivelli et al. (2004), Morduch (1990) en sus trabajos sobre América, de Dercon (1998) en sus análisis sobre África y Fafchamps y Pender (1997) sobre la India, donde analizan como el agricultor tradicional, debido a su limitada capacidad para afrontar disminuciones en la renta de sus explotaciones como consecuencias de catástrofes, fundamentalmente climáticas (Figura I) optan por una agricultura menos arriesgada y también menos rentable.

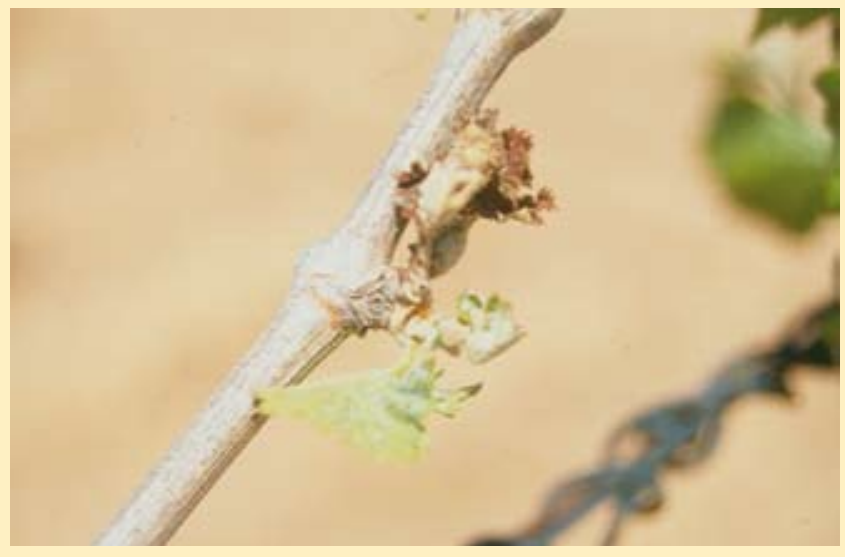

Figura I. Helada en brote de vid. Fuente: Santiago Escribano Pintor

\section{I.I La situación de partida.}

La actual política de seguros agrarios en España se inicia con la Ley 87//978, del 28 de diciembre, de Seguros Agrarios Combinados, en un clima de transición democrática. Aunque dentro del mundo agrario el camino a recorrer en relación con el resto de sectores productivos era mayor y por tanto la había que recorrer con una mayor aceleración para evitar las marginaciones que a lo largo de los tiempos se han ido produciendo en la agricultura española en este sentido. Serían esenciales las remodelaciones de Gobierno producidas en marzo de dicho año con la incorporación a la cartera de Agricultura de Jaime Lamo de Espinosa con el propósito fundamental de situar la vida rural en un lugar más digno aunque en muchas ocasiones dentro de la ignorancia por parte de otros sectores hacia la propia idiosincrasia del medio rural, siendo éste uno de los mayores lastres con que contaría la administración agraria durante esta época de transición, siendo también la causante de muchos de los conflictos que se generaron (Posada, 1983). Incluso en los Pactos de la Moncloa llegarían a tratarse materias agrarias por parte de las instancias firmantes con la pretensión de que la Agricultura alcanzara mayores cotas de protagonismo y no fuera como siempre apartada y marginada de la vida política, sin olvidar tampoco que en dichas fechas también se produjeron numerosas manifestaciones por parte de los agricultores a través de movilizaciones, conocidas como tractoradas, heredadas fundamentalmente del anterior régimen. Aunque quizás lo más destacable de aquella época en que se generó la norma fundamental de los seguros agrarios actuales es el clima de negociación que imprimió el nuevo ministro y que conducía a un nuevo estilo de relacionarse los agricultores con la Administración y viceversa.

La necesidad de la existencia de un seguro agrario eficaz es fundamental por el hecho de la permanencia de los ciclos productivos, es decir, en el hecho de que la producción agrícola poco transformada por las innovaciones técnicas sigue dependiendo actualmente de ciclos naturales, de tormentas, sequías y heladas (Sánchez Jiménez, 1975). La imposibilidad de modificar de forma apreciable el ciclo productivo de 
los cultivos principales en la mayoría de las zonas rurales españolas, como son, fundamentalmente, los cereales y la vid, deja a estas cosechas a disponibilidad de los agentes climatológicos incontrolables durante mayor tiempo, con lo que también el riesgo es mayor. Ello implica que el agricultor actual, al igual que sus antepasados, siga mirando hacia el cielo y hacia los boletines informativos meteorológicos, pero aún así, no habría que caer en lo que según Sánchez Jiménez indicaba, fundamentalmente, la preocupación por impedir que otros factores variables, controlables y fácilmente cambiantes, aparezcan con todo su vigor, de tal modo que como Flores de Lemus manifestaba, que las cosechas quedaran al cuidado de la Providencia divina, que es normalmente la que ha venido supliendo en nuestra historia a la previsión del Gobierno.

\section{I.2 El seguro agrario como instrumento de política agraria para la garantía de rentas.}

Para valorar la importancia del seguro agrario en España como elemento de garantía de rentas, debe recordarse que mediante el mismo los agricultores ven reducida su exposición a los riesgos productivos y económicos ajenos a su propio control, lo cual permite que el flujo de las rentas generadas en la explotación sea más estable, a pesar de las disminuciones en los rendimientos de los cultivos de su explotación, y se reduzca la probabilidad de quiebra incrementando la solvencia de la empresa agraria. Además, es indudable la mejora que supone en la gestión presupuestaria, ya que los procesos de valoración de los daños y de pago de las indemnizaciones los realizan las entidades aseguradoras de acuerdo con criterios preestablecidos normalizados, lo que permite que se evalúen los daños y se paguen las indemnizaciones en menos de sesenta días.

Pero los beneficios de la existencia de un buen sistema de seguros agrarios no sólo se encuentran a nivel de la explotación agrícola, sino también a nivel comarcal y ámbitos superiores, ya que al verse disminuida la producción regional, repercute en la productividad económica y en el resto de sectores económicos, con las consiguientes tensiones y des- equilibrios, y la consiguiente probabilidad de traducirse en una disminución de la calidad de vida del medio rural, así como en migraciones del campo al medio urbano. Lo que no cabe duda, es que como Dueñas Merino (2000) expresa:"la suscripción del seguro tiene que verse como un acto de solidaridad con el sector productor, pero no considerándose un elemento generador de rentas, sino como garante de estabilidad ante siniestros y por ello como un instrumento de política agraria y de interés público, al servicio del sector agropecuario y por extensión de toda la sociedad española".

Además, mediante el Seguro Agrario se favorece la creación de riqueza en general al evitar que los productores deban emplear recursos económicos en aminorar sus riesgos, al poder conseguirlo mediante el seguro a un coste menor, por la capacidad del sistema de compensar las pérdidas entre riesgos, zonas y agricultores.

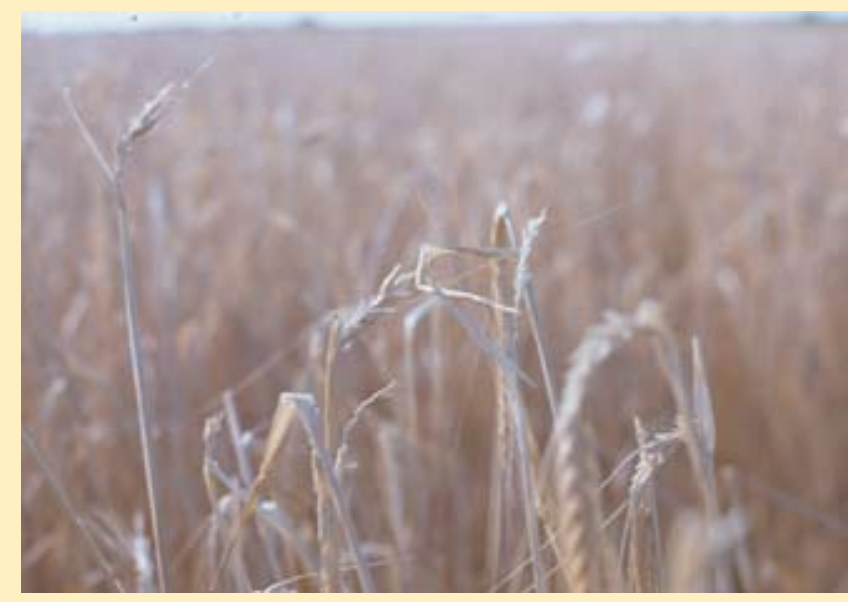

Figura 2. Siniestro de pedrisco sobre cebada. Fuente: Santiago Escribano Pintor.

Pero la importancia del hecho mencionado, e incluso de la razón de ser del seguro agrario, no sólo se debe valorar desde una perspectiva teórica, donde ya existen numerosos estudios que tratan la relación e importancia del seguro agrario con respecto a la agricultura tradicional, caracterizada por su limitada capacidad de innovación. En este escenario, si no fuera por la existencia de los seguros agrarios, y debido a su limitada capacidad para protegerse de dichos siniestros (Figura 2) así como para manejar de una forma eficiente los riesgos y siniestros, se llegaría a 
una situación que provocaría que los efectos de dichos siniestros inesperados tiendan a empobrecer $y$ a incrementar la vulnerabilidad de dichos agricultores de forma continua.

\section{Materiales y métodos.}

Procederemos a un análisis empírico en el ámbito español que llevaremos a cabo a través de las macromagnitudes agrarias, como fiel reflejo de la dimensión económica del sector.

De los tres indicadores más importantes de las macromagnitudes agrarias: el Valor Añadido Bruto a precios básicos (VAB), la renta real por unidad de trabajo (conocida como indicador $A$ en la nomenclatura de Eurostat) y la Renta Agraria o el valor añadido neto a coste de los factores, que se refiere a alValor Añadido Neto a precios básicos el importe de las otras subvenciones a la producción netas de impuestos. Mide la remuneración de todos los factores de producción (tierra, capital y trabajo) y puede denominarse Renta de los Factores ya que representa la totalidad del valor generado por una unidad dedicada a una actividad de producción. Utilizaremos este último para, a través de su evolución así como la de las indemnizaciones pagadas por el seguro agrario, contrastar la hipótesis por la que se constata la importancia del seguro agrario en su contribución al mantenimiento y mejora de la agricultura, al evitar la incertidumbre de las condiciones meteorológicas sobre los cultivos de las explotaciones y por tanto la garantía de la renta de los agricultores.

Por otro lado tendremos en cuenta los datos facilitados por la agrupación Agroseguro, S.A. que es una empresa mercantil española; donde se encuentran en régimen de pool la mayoría de las empresas aseguradoras española, encargada de gestionar el sistema de seguros agrarios en España. referentes a las indemnizaciones pagadas para el conjunto de las líneas que componen el seguro agrario en España.

La importancia relativa del sector agroalimentario respecto al $\mathrm{VAB}$ del total de la economía durante el último decenio se ha reducido sensiblemente al pasar de un 7,8\% en el año 1995 al 5,5\% del año 2005 (Agrlnfo, 2006). Aunque dicho descenso se debe al crecimiento superior en el resto de sectores, ya que aunque la Renta Agraria nominal perdió en año 2005 un $8,9 \%$, sin embargo sí que presenta una tendencia creciente en la última década con un aumento acumulado del $45 \%$, aunque en valores de Renta Agraria real tan sólo lo haga en un $2,7 \%$.

Otro aspecto a tener en cuenta para determinar si los seguros agrarios cumplen el objetivo de garantizar la estabilidad de las rentas, se basa según Compés et al. (200l) en el mayor o menor peso que tengan los rendimientos de un determinado cultivo para un determinado ámbito; y los precios que normalmente se fijan en ámbitos nacionales e internacionales. De tal modo que si no tienen una alta correlación negativa, el seguro reduce la variabilidad de los rendimientos pero no garantizaría en un alto grado la estabilidad de las rentas, por otro lado, en aquellos casos donde la correlación es muy negativa, es decir, precios muy altos cuando la cosecha es muy escasa, el seguro puede llegar incluso a sobreproteger los ingresos. Por lo que cuando se trata de siniestros a gran escala, como normalmente son las heladas y sequías, el seguro cumple con mayor eficacia el objetivo de garantizar las rentas, pues además de recibir la indemnización correspondiente, debido a la escasez de dicho producto en el mercado se produce una elevación de los precios que repercute de forma positiva en la renta de los agricultores. Aspecto que no tiene por qué cumplirse en el caso de siniestros más puntuales, como suele suceder con los pedriscos, que son mucho más limitados en extensión.

Si tenemos en cuenta los últimos seis años, podemos comprobar como la Renta Agraria tiene un comportamiento desigual a lo largo de los mismos, como se puede observar en el siguiente gráfico (Figura 3). Por lo que se ha preferido calcular la media móvil de los tres últimos años de la misma para cada año, la cual refleja una tendencia moderada al crecimiento. De este modo, comprobamos dentro del último decenio las campañas de los años 1999, 2002 y 2005 son aquellas en que la Renta Agraria sufrió un descenso si la comparamos con la media móvil de los últimos tres años, consecuencia de las adversidades meteorológicas que se manifestaron en una disminución de la producción agrícola y por tanto de la renta de las explotaciones agrarias. 


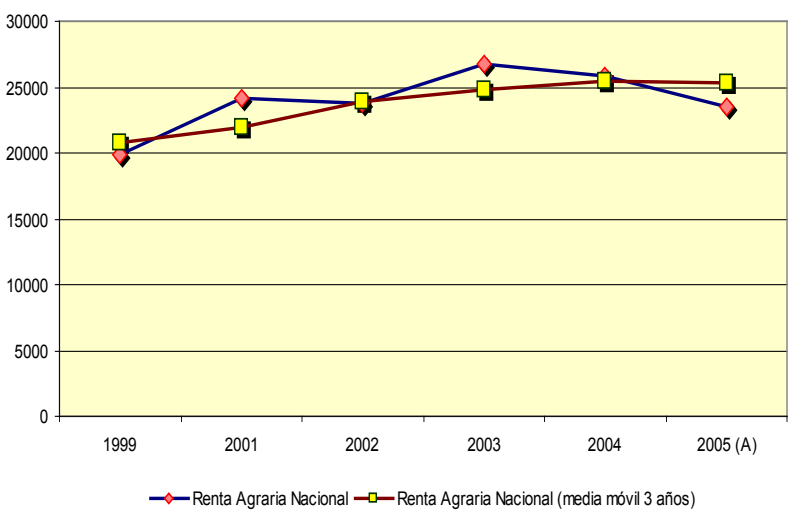

Figura 3. Evolución de la Renta Agraria Nacional y la Renta Agraria Nacional (media móvil de tres años) en España durante el periodo 1999-2005. Fuente: elaboración propia a partir de los datos del MAPA.

Las indemnizaciones desembolsadas por los seguros agrarios como consecuencia de las siniestros, fundamentalmente de índole meteorológica, han representado un comportamiento desigual a lo largo de los últimos años dependiendo de las campañas, desde el $0,92 \%$ del año 2003 al máximo del 2,45\% de la última campaña, observándose en el siguiente gráfico (Figura 4) la tendencia ascendente en cuanto a la participación de las indemnizaciones procedentes de los seguros agrarios sobre el total de Renta Agraria, lo que viene a confirmar el papel de actor principal que está adquiriendo esta herramienta de política agraria y social en el sector, medido desde un punto de vista estrictamente económico.

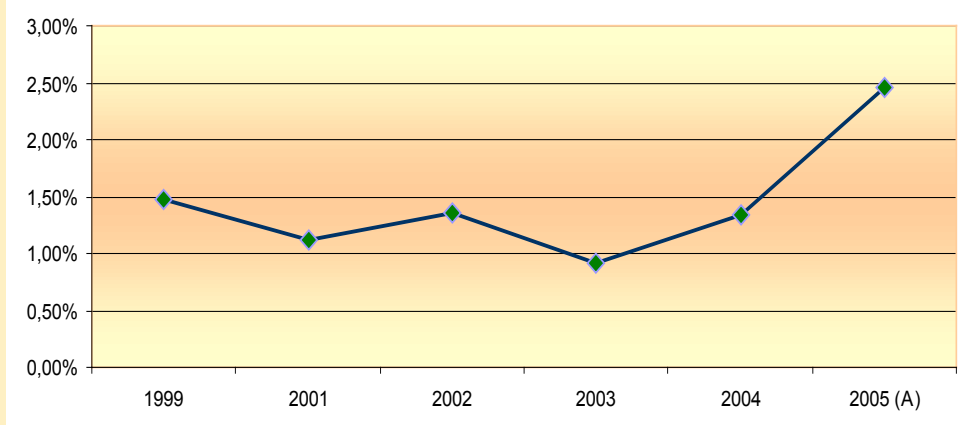

Figura 4. Evolución del porcentaje de participación sobre la Renta Agraria de las indemnizaciones desembolsadas por el sistema de seguros agrarios en el periodo 1999. 2005. Fuente: elaboración propia a partir de los datos del MAPA.
Pero, como se enunció anteriormente, para un correcto análisis de los datos disponibles utilizaremos las medias móviles, ya que con ella se consigue suavizar las series de datos sobre las que se calcula, eliminando la dificultad que plantea la erraticidad en las series anuales como consecuencia tanto de la disminución de renta como de las indemnizaciones como consecuencia de siniestros indemnizables, permitiendo observar de una forma más clara la dirección actual, es decir, la tendencia. Se trata de la misma metodología utilizada por Agrinfo (2006) y Burgaz (2006) para conseguir un análisis significativo de la valoración del seguro agrario como instrumento de estabilización de la renta de nuestros agricultores. También se debe tener en cuenta que las indemnizaciones derivadas del sistema de seguros agrarios no están incluidas en la Renta Agraria.

\section{Resultados}

Se realiza el análisis a nivel nacional y para el conjunto de las líneas de seguros agrarios. Por lo que se trata de comparar la desviación de la Renta Agraria Nominal en los años 1992, 1999 y 2005 respecto de la media móvil de los últimos tres años de la misma, así como la desviación de las indemnizaciones pagadas como consecuencia de siniestros agrarios indemnizables respecto de la media móvil de tres años sobre dichas indemnizaciones en los mismos periodos. Los resultados nos llevan a afirmar que las indemnizaciones pagadas por los seguros agrarios han contribuido a paliar la caída de la Renta Agraria en dichos años en porcentajes significativos, como son el hecho de atenuar la desviación de 91 I,38 millones de euros de la Renta para el año 1992 en más de un 7\%. Así como para el año 1999 se compensó en más de un 16\% la caída de 958,50 millones de euros en la renta de los agricultores, y en más del 10\% sobre los $1.581,32$ millones de euros de desviación de la Renta Agraria para el reciente año 2005 (Tabla I). 
Tabla I. Principales inidicadores en los años 1992, 1999 y 2005. Fuente: elaboración propia a partir de los datos del MAPA y Agroseguro, S.A.

\begin{tabular}{|c|c|c|c|}
\hline Años & 1992 & 1999 & 2005 \\
\hline I. Renta Agraria Nacional & 12374,32 & 19833,78 & 23470,72 \\
\hline $\begin{array}{l}\text { Il. Renta Agraria Nacional } \\
\text { (media móvil } 3 \text { años) }\end{array}$ & 13285,70 & 20792,29 & 25322,04 \\
\hline III. Desviación de la Renta (I-II) & $-911,38$ & $-958,50$ & $-|85|, 32$ \\
\hline IV. Indemnizaciones Seguros Agrarios & 237,69 & 293,00 & $\mathbf{5 7 5 , 0 0}$ \\
\hline $\begin{array}{l}\text { V. Indemnizaciones Seguros Agrarios } \\
\text { (media móvil } 3 \text { años) }\end{array}$ & $|69,5|$ & 139,00 & 388,33 \\
\hline VI. Desviación de indemnizaciones (IV-V) & 68,18 & 154,00 & 186,67 \\
\hline $\begin{array}{l}\text { VII. Porcentaje de las indemnizaciones } \\
\text { sobre Renta Agraria }\end{array}$ & $1,92 \%$ & $\mathrm{I}, \mathbf{4 8} \%$ & $2,45 \%$ \\
\hline $\begin{array}{l}\text { VIII. Porcentaje de indemnizaciones } \\
\text { en Renta Agraria (móvil) (VI/III) }\end{array}$ & $-7,48 \%$ & $-16,07 \%$ & $-10,08 \%$ \\
\hline
\end{tabular}

Si realizamos los cálculos pero teniendo en cuenta la media móvil, tanto para la Renta Agraria Nacional como para las Indemnizaciones pagadas por los seguros agrarios, cada dos años, los resultados son más abrumadores, pues en este caso se observa como para el mismo periodo analizado, hay más años donde se producen desviaciones negativas en la Renta Agraria, aunque de menor cantidad, actuando el sistema de seguros agrarios atenuando dicha desviación de la Renta Agraria (Tabla 2).

Tabla 2. Media móvil de los años I99I a 2005. Fuente: elaboración propia a partir de los datos del MAPA y Agroseguro, S.A.

\begin{tabular}{|c|c|c|c|c|c|}
\hline Años & 1991 & 1992 & 1999 & 2002 & 2005 \\
\hline I. Renta Agraria Nacional & $13.7 \mid 7,07$ & I 2.374,32 & $19.833,78$ & $23.736,30$ & $23.470,72$ \\
\hline $\begin{array}{l}\text { Il. Renta Agraria Nacional } \\
\text { (media móvil } 2 \text { años) }\end{array}$ & | 3.74|,38 & | 3.045,69 & $20.658,90$ & $23.919,40$ & $24.635,82$ \\
\hline III. Desviación de la Renta (I-II) & $-24,32$ & $-671,37$ & $-825, I I$ & $-183,10$ & $-1.165,10$ \\
\hline IV. Indemnizaciones Seguros Agrarios & 146,57 & 237,69 & 293,00 & 322,00 & 575,00 \\
\hline $\begin{array}{l}\text { V. Indemnizaciones Seguros Agrarios } \\
\text { (media móvil } 2 \text { años) }\end{array}$ & 135,42 & | 92, I 3 & $2|5| \mid$, & 295,50 & 459,50 \\
\hline $\begin{array}{l}\text { VI. Desviación de indemnizaciones } \\
\text { (IV-V) }\end{array}$ & 11,16 & 45,56 & 77,89 & 26,50 & I I 5,50 \\
\hline $\begin{array}{l}\text { VII. Porcentaje de indemnizaciones } \\
\text { en Renta Agraria (móvil) (VI/III) }\end{array}$ & $-45,88 \%$ & $-6,79 \%$ & $-9,44 \%$ & $-14,47 \%$ & $-9,91 \%$ \\
\hline
\end{tabular}

\section{Conclusiones}

De los análisis anteriores, a pesar de que el seguro agrario no se encuentra plenamente implantado en todos los ámbitos y zonas de forma homogénea, nos permiten afirmar la importancia del actual sistema de seguros agrarios como elemento de compensación de la Renta Agraria cuando cae la misma como consecuencia de siniestros meteorológicos, tal y como se aprecia en el cálculo de la desviación de dicha Renta 
Agraria tanto al aplicar medias móviles de dos como de tres años. En todo caso, hay que tener en cuenta, que dichas compensaciones no pueden ni debe pretenderse que aparezcan todos los años, ya que entonces dejaría de ser propiamente la institución del seguro agrario para convertirse en un elemento generador de rentas. Ese es el motivo por el que solamente se muestra el efecto positivo del seguro agrario en determinadas campañas.

El seguro agrario como tal, no debe considerarse como una política agraria sustituta de otro tipo de herramientas de política agraria, ni de aquellas que pretenden fomentar la productividad, ni tam- poco considerarse como un elemento generador de rentas. El seguro agrario tiene su especificidad propia, y si se quiere mantener su solvencia y estabilidad en el tiempo no puede usarse para otro tipo de objetivos que los realmente le caracterizan como tal.

De lo que no hay duda es que en la situación actual nos encontramos con el sistema de protección más evolucionado del último siglo que se ha conseguido en España, basado en su carácter semi-publico donde la administración no gestiona directamente, aunque mantiene el poder de algunas funciones importantes dentro del sistema.

\section{Referencias}

Burgaz, F. J. 2006. Pasado y presente de los seguros agrarios: lecciones aprendidas $y$ futuros desarrollos. Conferencia Internacional EI Seguro Agrario como instrumento para la gestión de riesgos. Madrid, España.

Compés López, R. García Álvarez-Coque, J. M. y Martínez Gómez, V. 200I. Viabilidad de los seguros de ingresos en la agricultura española: el fondo de la patata de Álava. Pamplona: IV Congreso Nacional de Economía Agraria dentro del Área Temática Políticas Agrarias y Globalización.

Dercon, S. 1998. Wealth, Risk and Activity Choice: Cattle in Western Tanzania. Journal of Development Economics. (55).

Dueñas Merino, G. 2000. Un seguro agrario para el siglo XXI. Noticias del Seguro Agrario. (12) Pp. 8-9

Fafchamps, M. y Pender, J. 1997. Precautionary Saving, Credit Constraints, and Irreversible Investment: Theory and Evidence from semi-arid India. Journal of Business and Economic Statistics. (15,2) Pp. I80-194.

López de Coca, N. 1997. Un factor de estabilidad en el medio rural. Vida Rural. Suplemento. $\mathrm{n}^{\circ}$ 47, pp. 7

Morduch, J. 1990. Risk, Production and Savings: Theory and Evidence from Indian Households. Harvard University.

Posada, L. J. 1983. La política agraria durante la Transición democrática. Papeles de Economía Española, $n^{\circ} 16$ pp 3।3-32।

Sánchez Jiménez,J. I 975. La vida rural en la España del siglo XX. Madrid: Ed. Planeta.

Trivelli, C. Escobal, J. y Revesz, B. 2004. Red de desarrollo rural: alternativas para la pequeña agricultura comercial. Lima: Propuesta de red de investigación presentada al CIES.

Unidad de Análisis y Prospectiva. SG de Planificación Económica y Coordinación Institucional. 2006. Renta Agraria. AgrInfo. 5, pp. I-2 